\title{
Optimal Portfolio Selection of Mean-Variance Utility with Stochastic Interest Rate
}

\author{
Shuang Li, ${ }^{1}$ Shican Liu, ${ }^{2}$ Yanli Zhou $\mathbb{D}^{3}{ }^{3}$ Yonghong Wu, ${ }^{4}$ and Xiangyu Ge $\mathbb{D}^{2}$ \\ ${ }^{1}$ School of Mathematics and Physics, Mianyang Teachers' College, Mianyang 621000, China \\ ${ }^{2}$ School of Statistics and Mathematics, Zhongnan University of Economics and Law, Wuhan 430073, China \\ ${ }^{3}$ School of Finance, Zhongnan University of Economics and Law, Wuhan 430073, China \\ ${ }^{4}$ Department of Mathematics and Statistics, Curtin University, Perth, WA 6102, Australia \\ Correspondence should be addressed to Xiangyu Ge; xiangyu_ge@163.com
}

Received 21 July 2020; Revised 26 August 2020; Accepted 20 October 2020; Published 19 November 2020

Academic Editor: Yuanfang Ru

Copyright (c) 2020 Shuang $\mathrm{Li}$ et al. This is an open access article distributed under the Creative Commons Attribution License, which permits unrestricted use, distribution, and reproduction in any medium, provided the original work is properly cited.

In order to tackle the problem of how investors in financial markets allocate wealth to stochastic interest rate governed by a nested stochastic differential equations (SDEs), this paper employs the Nash equilibrium theory of the subgame perfect equilibrium strategy and propose an extended Hamilton-Jacobi-Bellman (HJB) equation to analyses the optimal control over the financial system involving stochastic interest rate and state-dependent risk aversion (SDRA) mean-variance utility. By solving the corresponding nonlinear partial differential equations (PDEs) deduced from the extended HJB equation, the analytical solutions of the optimal investment strategies under time inconsistency are derived. Finally, the numerical examples provided are used to analyze how stochastic (short-term) interest rates and risk aversion affect the optimal control strategies to illustrate the validity of our results.

\section{Introduction}

Portfolio optimization, which is an important topic in the financial market, has been studied by a vast of researchers after the first publication by Markowitz [1]. In portfolio optimization from a given utility function and system parameters, the optimal values of the control parameters are determined to maximize the final utility. Previous research results in this field can be cataloged by the utilities used such as the mean-variance utility [2], the endogenous habit formation [3], the hyperbolic discounting [4], and the classic constant relative risk aversion (CRRA) [5]. Among those utilities, optimal asset allocation under mean-variance is one of the very interesting and thought-provoking topics in the classic results of financial economics. Under this framework, that optimal asset allocation problem within multiple periods is time-inconsistent which prohibits the application of the classical Bellman Optimality Principle. More precisely, the time inconsistency states that the optimal control depends on the starting point. This basically means that the control strat- egy that is optimal on the full-time interval $[0, T]$ may not be optimal in the subinterval of $[t, T]$. Currently, there are two widely used ways for handling this type of problem.

One common method is called the buy and hold of the precommitted strategy. From the point of economical meaning, Kydland and Prescott [6] explain this tactic. Richardson [7] is credited for being the first to study the portfolio optimization problem by using the mean-variance utility function in the continuous time. He was soon followed by BajeuxBesnainou and Portait [8]. They used a single stock with a constant risk-free rate for their studies. Using the same motivation, $\mathrm{Li}$ and $\mathrm{Ng}$ [9] change the classical mean-variance problem to a stochastic linear quadratic control one. Further research such as adding transaction cost, extending, and improving the models has been done by Dai et al. [10], Xue et al. [11], Lim [12], Bielecki et al. [13], Yao et al. [14], and Xia [15].

Recently, researchers have found that the volatility model is constructed with a stochastic model rather than a 
deterministic model (see Christoffersen et al. [16], Heston [17], Li et al. [18], Liu et al. [19]). Commonly used optimal asset allocation strategies are mostly controlled by the Hamilton-Jacobi-Bellman (HJB) equation. A more intense way of analysing the time-inconsistent problem has been to put it in a game theoretic context with the aim of making the model more realistic. In brief, the control process under a multiperiod time is like a game where the periods are treated as multiple players. Each player presents the accordingly current flavour and the incarnations of future tastes. The game ends when we find the subgame perfect Nash equilibrium point. As mentioned already, Markowitz [1] was the pioneer of this field, and he can also be credited with introducing the game theory approach to tackle the meanvariance portfolio optimization problem. Other researchers who also used this approach are Vieille and Weibull [20], Goldman [21], Peleg and Yaar [22], Pollak [23], Wu and Zhuang [24], and Krusell and Smith [25]. Basak and Chabakauri [26], especially, were interested in using the game theory for the continuous portfolio optimization problem. And the development, both from the modeling and the actual economic meaning, is made by Bjork and Murgoci [27] through extending the analysis to other different objective functions than the mean-variance one. Along with the work of Bjork et al. [4], Bjork and Murgoci [27] study the mean-variance portfolio optimization by relaxing the CRRA into a dynamic one which depends on the current wealth which is more economically reasonable.

In this paper, we follow the work of Bjork et al. [4], with the aim of making a further realistic financial model. We achieve this by relaxing the assumption of a constant interest rate and converting it to a stochastic (short-term) interest rate that is nested by the shown SDE [28]:

$$
d r_{t}=\left(\alpha+\beta r_{t}\right) d t+\varepsilon \eta^{\theta} d B_{t}^{r}
$$

The parameters in the stochastic processes are determined through the classical experimental data. For this particular problem, we then present the generalized extension of the HJB equation, applying the developed control theory with time inconsistency by Bjork and Murgoci [27]. Finally, we will proceed to provide a numerical illustration to show our results.

The remaining of the article is compiled in the following manner: in Section 2, we explain the setting of the financial market, while the structure is of a mean-variance optimal asset portfolio model with the stochastic (short-term) interest rate under state-dependent risk aversion (SDRA). Also, an extension of the HJB equation is derived along with the stochastic (short-term) interest rate. In Section 3, a closedform analytical solution is given for the optimal investment strategy across a number of situations. The numerical study is presented in Section 4 with some comparisons, and a conclusion is given in Section 5.

\section{The Basic Framework}

2.1. The Model. Let the model be composed of two assets being a stock and a bond with the interest rate of the bond being governed by a stochastic process. Then, the bond $F_{t}$ will follow the process described in the following SDE

$$
d F_{t}=F_{t} r_{t} d t, F(0)=1
$$

and the stock price $S_{t}$ and stochastic interest rate $r_{t}$ are modeled by the following stochastic process,

$$
\begin{aligned}
& d S_{t}=\mu S_{t} d t+\sigma S_{t} d B_{t}^{s}, \\
& d r_{t}=\left(\alpha+\beta r_{t}\right) d t+\varepsilon \eta^{\theta} d B_{t}^{r},
\end{aligned}
$$

with the parameter $\mu$ as the drift rate of $S_{t}$ and $\sigma$ as the volatility of $S_{t}$. We assume that the Brownian motion $\left(B_{t}^{r}, B_{t}^{s}\right)$ is correlated with the correlation $\operatorname{Cov}\left(B_{t}^{r}, B_{t}^{s}\right)=\rho_{s r}$. The stochastic term structure model of interest rate, both single factor and multifactor, is nested within the above SDE (4). If we choose the most suitable restrictions on the six parameters $\alpha, \beta, \varepsilon, \theta, \eta$, and $\rho_{s r}$, then the SDE in (4) can be used to describe an array of interest rate processes of which some commonly known ones include Merton [29], Vasicek [30], and the CIR model [31].

Here, the total wealth is given as $W_{t}$, while the initial wealth is $W_{0}$, and as mentioned above, we apportion this wealth to the two asset classes. The amount of money we invest in the stock $S_{t}$ is denoted by $u_{t}$, and the rest will be invested in the bond. Thus, we have the stochastic process of the total wealth

$$
d W_{t}=\left[r_{t} W_{t}+\left(\mu-r_{t}\right) u_{t}\right] d t+\sigma u_{t} d B_{t}^{s}
$$

2.2. The Optimal Asset Portfolio Selection Model. Let $\mathbb{U}[0, T]$ $=\left\{u_{t}:[0, T] \rightarrow \mathbb{R}\right\}$ be the set of all possible acceptable control strategies in the time period $[0, T]$. Then, we define the mean-variance portfolio selection problem as an optimal solution for the most likely strategy that the mean-variance utility is maximal at the termination period $T$. Also, the mean-variance utility has risk aversion that depends on wealth shown by $\gamma(w)$. So we have the objective function $J$ of time $t$, interest rate $r$, wealth $w$, and the optimal strategy $u$ described as

$$
J(t, r, w, u)=E\left[W_{T}\right]-\frac{\gamma(w)}{2} \operatorname{Var}\left[W_{T}\right]
$$

The goal is to solve for the most optimal admissible strategy $\widehat{u}$ such that the mean-variance utility function reaches its maximal at the termination time $T$. Simply put, we want to optimize our expected returns even when there is a risk involved. Thus, we have the equation

$$
\begin{aligned}
V(t, r, w) & =\max _{u \in U}\left\{E_{t, r, w}\left[W_{T}^{U}\right]-\frac{\gamma(w)}{2} \operatorname{Var}_{t, r, w}\left[W_{T}^{U}\right]\right\} \\
& =J(t, r, w, \widehat{u}) .
\end{aligned}
$$


We could rewrite $J(t, r, w, u)$ as

$$
J(t, r, w, u)=E_{t, r, w}\left[F\left(w, W^{\mathbf{u}}(T)\right)\right]+G\left(w, E_{t, r, w}\left[W^{\mathbf{u}}(T)\right]\right),
$$

where

$$
F(w, y)=y-\frac{\gamma(w)}{2} y^{2}, G(w, y)=\frac{\gamma(w)}{2} y^{2}
$$

2.3. The Game Theoretic Framework. There are various approaches for solving time-inconsistent problems. One of the most suited methods is to use the subgame perfect Nash equilibrium theory by Bjork et al. [4], especially considering our problem. The brief explanation of the theory is as follows: suppose that we have several players and each player will have a control $u$. If at the later time point $t$ the player chooses the optimal control $u(\cdot, t)$, we simply regard that the player at time $l, l \leq t$, will still use this control $u(\cdot, l)$ as the optimal one. Using this theory, then we can formally provide a mathematical equation for the formal equilibrium control as the following equation

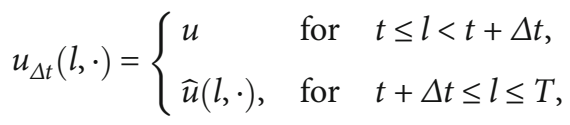

with $(t, r, w) \in[0, T] \times R^{n} \times R^{n}$ and $u \in R^{k}, \Delta t>0$.

When the condition

$$
\lim _{\Delta t \rightarrow 0} \inf \frac{J(t, r, w, \widehat{u})-J\left(t, r, w, u_{\Delta t}\right)}{\Delta t} \geq 0,
$$

for $(t, r, w) \in[0, T] \times R^{n} \times R^{n}$ and $u \in R^{k}$ are satisfied, we can define the $\widehat{u}$ as the equilibrium control, and hence, we get the equilibrium value function in the form of (7).

Because the traditional Bellman optimality principle cannot be used to solve the optimal control problem with time inconsistency, the extended HJB equations can be formulated to solve this problem.

Definition 1. Let $A$ be an infinitesimal generator; then, for any fixed control $u \in U$, the associate infinitesimal generator is denoted as $A^{u}$ and thus is defined as follows

$$
A^{u}=\frac{\partial}{\partial t}+a_{1} \frac{\partial}{\partial w}+b_{1} \frac{\partial}{\partial r}+a_{2} \frac{\partial^{2}}{\partial w^{2}}+b_{2} \frac{\partial^{2}}{\partial r^{2}}+c \frac{\partial^{2}}{\partial w \partial r}
$$

where

$$
\begin{aligned}
& a_{1}=r_{t} w_{t}+\left(\mu-r_{t}\right) u, b_{1}=\alpha+\beta r_{t}, a_{2}=\frac{1}{2} \sigma^{2} u^{2}, \\
& b_{2}=\frac{1}{2} \varepsilon^{2} \eta^{2 \theta}, c=\varepsilon \eta^{\theta} \sigma u \rho_{s r} .
\end{aligned}
$$

From the analysis of Bjork and Murgoci [27], we define the following extended HJB equations for the Nash equilibrium problem with a stochastic interest rate.

\section{Theorem 2.}

$$
\begin{aligned}
& \sup _{u \in U}\left\{A^{u} V(t, r, w)-A^{u} f(t, r, w, w)+A^{u} f^{w}(t, r, w)\right. \\
& \left.-A^{u}(G * g)(t, r, w, w,)+H^{u} g(t, r, w, w)\right\}=0,0 \leq t \leq T,
\end{aligned}
$$

has the following boundary conditions

$$
\begin{aligned}
A^{\widehat{u}} f^{y}(t, r, w) & =0, A^{\widehat{u}} g(t, r, w)=0,0 \leq t \leq T, \\
V(T, r, w) & =F(w, w)+G(w, w), f(T, r, w, y) \\
& =F(y, w), f(t, r, w, y)=f^{y}(t, r, w), \\
g(T, r, w) & =w,(G * g)(t, r, w)=G(w, g(t, r, w)), \\
H^{u} g(t, r, w, w) & =G_{y}(w, g(t, r, w)) \dot{A}^{u} g(t, r, w), G_{y}(w, y)=\frac{\partial G}{\partial y}(w, y) .
\end{aligned}
$$

We derived Theorem 2 above, please see Appendix.

Remark 3. In the above theorem, $f(\cdot)$ represents $f(t, r, w, y)$ and $f^{y}(\cdot)$ represents $f^{y}(t, r, w)$. Function $f(\cdot)$ is not the same as $f^{y}(\cdot) . f(\cdot)$ is the function of the four variables $t, \mathrm{r}, w, y$, while $f^{y}(\cdot)$ is a function of the three variables $t, r, w$. But if the parameters $y$ are fixed, $f(t, r, w, y)$ is equal to $f^{y}(t, r, w)$.

\section{The Main Results}

In this paper, we describe risk aversion as the willingness to accept the loss (or profit) when exposed to uncertainty in the future. A risk-averse investor will be more cautious about their wealth when the payoff at a later date is unknown. Such a person will opt to place their wealth in a low-incomegenerating investment, for instance, a checkable bank account with high certainty rather than a high-incomegenerating stock with high uncertainty. We can have a constant risk-averse investor who bears the same investment attitude towards different scenarios at all times.

Earlier research suggests that authors assumed the risk aversion as a constant. This implies that the equilibrium solution of the control for the dollar-invested amount in the risky asset was independent on the investor's current wealth. We relax this assumption to be more economically realistic and impose a SDRA term. Further, we suggest the interest rate be regarded as a stochastic process so that it reflects the real economy rather than a constant rate with no relationship to other economic factors.

3.1. Mean-Variance Utility with Stochastic Interest Rate under SDRA. We proceed to give a more realistic equation for the stochastic process, where the SDRA $\gamma(w)$ along with a stochastic interest rate can give an investment strategy that depends on the current wealth. This is,

$$
J(t, r, w, U)=E_{t, r, w}\left[W_{T}^{U}\right]-\frac{\gamma(w)}{2} \operatorname{Var}_{t, r, w}\left[W_{T}^{U}\right]
$$

According to the extended HJB equation (14) in 
Theorem 2, we have

$$
\begin{aligned}
V_{t} & +\sup _{u \in U}\left\{a_{1} V_{w}-b_{1} V_{r}+a_{2} V_{w w}+b_{2} V_{r r}+c V_{w r}\right. \\
& -a_{1} f_{y}-a_{2} f_{y y}-2 a_{2} f_{w y}-c f_{y r}-a_{1} G_{w}-a_{2} G_{w w} \\
& \left.+2 G_{w y} g_{w}+G_{y y} g_{w}^{2}-b_{2} G_{y y} g_{r}^{2}-c G_{w y} g_{r}+G_{y y} g_{r} g_{w}\right\}=0,
\end{aligned}
$$

$$
A^{\widehat{u}} f^{y}(t, r, w)=0, A^{\widehat{u}} g(t, r, w)=0,0 \leq t \leq T \text {. }
$$

After the simplification (17), we have

$$
\begin{aligned}
& V_{t}+\sup _{u \in U}\left\{\left(r_{t} w_{t}+\left(\mu-r_{t}\right) u_{t}\right)\left[V_{w}-f_{y}-G_{w}\right]\right. \\
& +\left(\alpha+\beta r_{t}\right) V_{r}+\frac{1}{2} \sigma^{2} u^{2}\left[V_{w w}-f_{y y}-2 f_{w y}-G_{w w}\right. \\
& \left.\quad-2 G_{w y} g_{w}-G_{y y} g_{w}^{2}\right]+\frac{1}{2} \varepsilon^{2} \eta^{2 \theta}\left[V_{r r}-G_{y y} g_{r}^{2}\right] \\
& \left.+\varepsilon \eta^{\theta} \sigma u \rho_{s r}\left[V_{w r}-f_{y r}-G_{w y} g_{r}-G_{y y} g_{r} g_{w}\right]\right\}=0, \\
& f_{t}+\left(r_{t} w_{t}+\left(\mu-r_{t}\right) \widehat{u}\right)\left[f_{w}\right]+\left(\alpha+\beta r_{t}\right) f_{r}+\frac{1}{2} \sigma^{2} u^{2} f_{w w} \\
& \quad+\frac{1}{2} \varepsilon^{2} \eta^{2 \theta} f_{r r}+\varepsilon \eta^{\theta} \sigma u \rho_{s r} f_{w r}=0, \\
& \quad+\frac{1}{2} \varepsilon^{2} \eta^{2 \theta} g_{r r}+\varepsilon \eta^{\theta} \sigma u \rho_{s r} g_{w r}=0,
\end{aligned}
$$

For the purpose of completeness, we recall the dynamics processes,

$$
\begin{aligned}
& V(t, r, w)=E_{t, r, w}\left[W_{T}^{\widehat{U}}\right]-\frac{\gamma(w)}{2} \operatorname{Var}_{t, r, w}\left[W_{T}^{\widehat{U}}\right] \\
& f(t, r, w, y)=E_{t, r, w}\left[W_{T}^{\widehat{U}}\right]-\frac{\gamma(y)}{2} E_{t, r, w}\left[\left(W_{T}^{\widehat{U}}\right)^{2}\right], \\
& g(t, r, w)=E_{t, r, w}\left[W_{T}^{\widehat{U}}\right] .
\end{aligned}
$$

From the above equations, we have

$$
\begin{gathered}
V_{t}=f_{t}+\gamma g g_{t}, V_{w}=f_{w}+f_{y}+\frac{\gamma_{w}}{2} g^{2}+\gamma g g_{w}, V_{r}=f_{r}+\gamma g g_{r}, \\
V_{w w}=f_{w w}+2 f_{w y}+f_{y y}+\frac{\gamma_{w w}}{2} g^{2}+2 \gamma_{w} g g_{w}+\gamma g_{w}^{2}+\gamma g g_{w w}, \\
V_{r r}=f_{r r}+\gamma g_{r}^{2}+\gamma g g_{r r}, V_{w r}=f_{w r}+f_{y r}+\gamma_{w} g g_{r}+\gamma g_{r} g_{w}+\gamma g g_{w r}, \\
G_{w}=\frac{\gamma_{w}}{2} y^{2}, G_{w w}=\frac{\gamma_{w w}}{2} y^{2}, G_{y}=\gamma y, G_{y y}=\gamma, G_{w y}=\gamma_{w} y .
\end{gathered}
$$

Substituting all the equations above to (19), we obtain

$$
\begin{aligned}
f_{t} & +\gamma g g_{t}+\sup _{u \in U}\left\{\left(r_{t} w_{t}+\left(\mu-r_{t}\right) u_{t}\right)\left[f_{w}+\gamma g g_{w}\right]\right. \\
& +\left(\alpha+\beta r_{t}\right)\left[f_{r}+\gamma g g_{r}\right]+\frac{1}{2} \sigma^{2} u^{2}\left[f_{w w}+\gamma g g_{w w}\right] \\
& \left.+\frac{1}{2} \varepsilon^{2} \eta^{2 \theta}\left[f_{r r}+\gamma g g_{r r}\right]+\varepsilon \eta^{\theta} \sigma u \rho_{s r}\left[f_{w r}+\gamma g g_{w r}\right]\right\}=0,
\end{aligned}
$$

or

$$
f_{t}+\gamma g g_{t}+\sup _{u} Z=0
$$

where

$$
\begin{aligned}
Z= & \left(r_{t} w_{t}+\left(\mu-r_{t}\right) u_{t}\right)\left[f_{w}+\gamma g g_{w}\right]+\left(\alpha+\beta r_{t}\right)\left[f_{r}+\gamma g g_{r}\right] \\
& +\frac{1}{2} \sigma^{2} u^{2}\left[f_{w w}+\gamma g g_{w w}\right]+\frac{1}{2} \varepsilon^{2} \eta^{2 \theta}\left[f_{r r}+\gamma g g_{r r}\right] \\
& +\varepsilon \eta^{\theta} \sigma u \rho_{s r}\left[f_{w r}+\gamma g g_{w r}\right] .
\end{aligned}
$$
that is

The optimization problem in (25) requires $d Z /\left.d u\right|_{\widehat{u}}=0$,

$$
\begin{aligned}
& \left.\frac{d Z}{d u}\right|_{\widehat{u}}=\left(\mu-r_{t}\right)\left(f_{w}+\gamma(w) g g_{w}\right)+\varepsilon \eta^{\theta} \sigma u \rho_{s r} \\
& \quad \cdot\left(f_{w r}+\gamma(w) g g_{w r}\right)+\sigma^{2}\left(f_{w w}+\gamma(w) g g_{w w}\right) \widehat{u}=0,
\end{aligned}
$$

By solving the above equation (27), we obtain the optimal control $\widehat{u}$ as

$\widehat{u}_{t}=-\frac{\left(\mu-r_{t}\right)\left(f_{w}+\gamma(w) g g_{w}\right)+\varepsilon \eta^{\theta} \sigma u \rho_{s r}\left(f_{w r}+\gamma(w) g g_{w r}\right)}{\sigma^{2}\left(f_{w w}+\gamma(w) g g_{w w}\right)}$.

Remark 4. If $\rho_{s r}=0, \eta=0$, the stochastic process of the bond becomes a risk-free bond. Thus, our result (28) reduces to

$$
\widehat{u}_{t}=-\frac{\left(f_{w}+\gamma(w) g g_{w}\right) \beta}{\left(f_{w w}+\gamma(w) g g_{w w}\right) \sigma^{2}}
$$

which is the same as Zhang et al. [32] if $\mu-r_{t}=\beta$.

3.2. Mean-Variance Utility with Stochastic Interest Rate under CRRA. Here, we relax the assumption to consider the case where the investor has the same risk tolerance all the time, but the interest rate is still stochastic. The wealth dynamic process is the same as (5) with the expected value function as follows

$$
\begin{gathered}
J(t, r, w, U)=E_{t, r, w}\left[F\left(W_{T}^{U}\right)\right]+G\left(E_{t, r, w}\left[W_{T}^{U}\right]\right), \\
F(w)=w-\frac{\gamma}{2} w^{2}, G(w)=\frac{\gamma}{2} w^{2}
\end{gathered}
$$


where

$$
\begin{gathered}
\sup _{u \in U}\left(A^{u} V\right)(t, r, w)=0, \\
V_{t}+\sup _{u \in U}\left\{a_{1} V_{w}-b_{1} V_{r}+a_{2} V_{w w}+b_{2} V_{r r}+c V_{w r}\right\}=0, \\
A^{\widehat{u}} f=0, A^{\widehat{u}} g=0, f(T, r, w)=w-\frac{\gamma}{2} w^{2}, g(T, r, w)=w .
\end{gathered}
$$

By using the same method such as (28), we have the optimal control as

$$
\widehat{u}_{t}=-\frac{\left(\mu-r_{t}\right)\left(f_{w}+\gamma g g_{w}\right)+\varepsilon \eta^{\theta} \sigma u \rho_{s r}\left(f_{w r}+\gamma g g_{w r}\right)}{\sigma^{2}\left(f_{w w}+\gamma g g_{w w}\right)} .
$$

3.3. Mean-Variance Utility with Constant Interest Rate under CRRA. We consider the simplest situation where both of the assumptions are removed. That is, the investor has the same risk tolerance all the time, and the interest rate does not change in the life of the whole investment. The dynamics of the wealth process are the same as (5) with the expected value function of $J(t, w, U)=E_{t, w}\left[F\left(W_{T}^{U}\right)\right]+$ $G\left(E_{t, w}\left[W_{T}^{U}\right]\right)$ in (9). Therefore, the optimal control can be shown as follows

$$
\widehat{u}_{t}=-\frac{(\mu-r)}{\sigma^{2} \gamma} e^{-r(T-t)} .
$$

\section{Numerical Study}

Using dimension analysis and applying the mean-variance utility function for the returns instead of the wealth itself, we naturally come up with the explicit form of the statedependent risk function. This model is called the "natural" risk function, which is expressed as $\gamma(w)=\gamma / w$. In an economic sense, that is to say that when the investor is more wealthy, the investor is more willing to invest in risky assets.

We can then rewrite the problem of the nonlinear PDE for the mean-variance portfolio optimization as

$$
\left\{\begin{array}{l}
A^{\widehat{u}} f(t, r, w, y)=0, \\
A^{\widehat{u}} g(t, r, w)=0,
\end{array}\right.
$$

with the operator

$$
\begin{gathered}
A^{u \wedge}=\frac{\partial}{\partial t}+a_{1} \frac{\partial}{\partial w}+b_{1} \frac{\partial}{\partial r}+a_{2} \frac{\partial^{2}}{\partial w^{2}}+b_{2} \frac{\partial^{2}}{\partial r^{2}}+c \frac{\partial^{2}}{\partial w \partial r}, \\
a_{1}=r_{t} w+\left(\mu-r_{t}\right) \widehat{u}, b_{1}=\alpha+\beta r_{t}, a_{2}=\frac{1}{2} \sigma^{2} \widehat{u}^{2}, \\
b_{2}=\frac{1}{2} \varepsilon^{2} \eta^{2 \theta}, c=\varepsilon \eta^{\theta} \sigma \widehat{u} \rho_{s r} .
\end{gathered}
$$

In order to solve (34) numerically by the finite difference method, we firstly assume that
TABLE 1: The value of parameters.

\begin{tabular}{lcc}
\hline Parameter & Symbol & Value \\
\hline Termination time & $T$ & 1 \\
Initial wealth & $w(0)$ & 1 \\
Risky asset appreciation rate & $\mu$ & 0.12 \\
Risky asset volatility & $\sigma$ & 0.2 \\
Interest restriction parameter & $\alpha$ & 0.189 \\
Interest restriction parameter & $\beta$ & -0.1612 \\
Interest restriction parameter & $\theta$ & 0 \\
Interest rate volatility & $\eta$ & 0.28 \\
Coefficient between two assets & $\rho_{s r}$ & -0.1 \\
\hline
\end{tabular}

$$
f(t, w, r, y)=a(t, r) w-\frac{\gamma}{2 y} b(t, r) w^{2}, g(t, r, w)=a(t, r) w
$$

Substituting (36) into the optimal control equation (28), we obtain $\widehat{u}=\tilde{u} w$ with the optimal control $u^{*}$ as

$$
u^{*}=\frac{\mu-r a-\gamma b+\gamma a^{2}}{\sigma^{2}}+\frac{\varepsilon \eta^{\theta} \sigma \rho_{s r}}{\sigma^{2}} \frac{a_{r}-\gamma b_{r}+\gamma a a_{r}}{\gamma b} .
$$

Thus, the two dimensional nonlinear PDEs reduce to a one dimension nonlinear PDEs

$$
\begin{gathered}
\dot{a}+\tilde{a}_{1} a+b_{1} a_{r}+b_{2} a_{r r}+\tilde{c} a_{r}=0, \\
\frac{1}{2} \dot{b}+\tilde{a}_{1} b+\frac{1}{2} b_{1} b_{r}+\frac{1}{2} b_{2} b_{r r}+\tilde{a}_{2} b+b_{r} \tilde{c}=0 .
\end{gathered}
$$

This problem can be solved numerically using the finite difference method. The nonlinear term is discretized by the fix-point iteration method. By using the above numerical method, we analyze

(i) The optimal investment strategy under different risk aversion

(ii) How the stochastic interest affects the optimal investment under different risk aversion

(iii) The total wealth processes under different statedependent values of risk aversion

Relaxing some assumptions, we can make some of the parameters being constant for the save of convenience while still preserving generality. The values of the parameters are provided in Table 1.

Figure 1 shows the optimal investment ratio $u^{*}$ starting from time $t=0$ to the end of the period $t=T$ under different CRRA values. During the investment period, the investor gradually reduces the investment in the risky assets over the long run under the different risk aversions. However, if the investor is less risk-averse, the investor will increase the investor's holdings in the risky assets. On the contrary, when risk aversion gets larger, the investment ratio of holding risky 


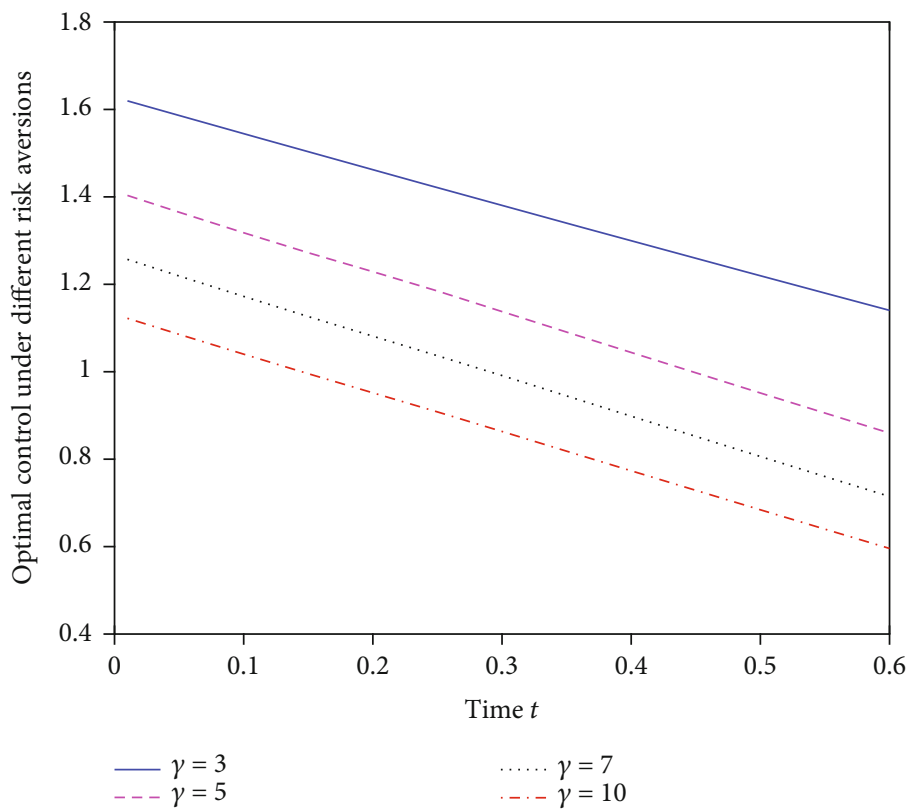

Figure 1: Optimal investment ratio $u^{*}$ with different $\gamma$.

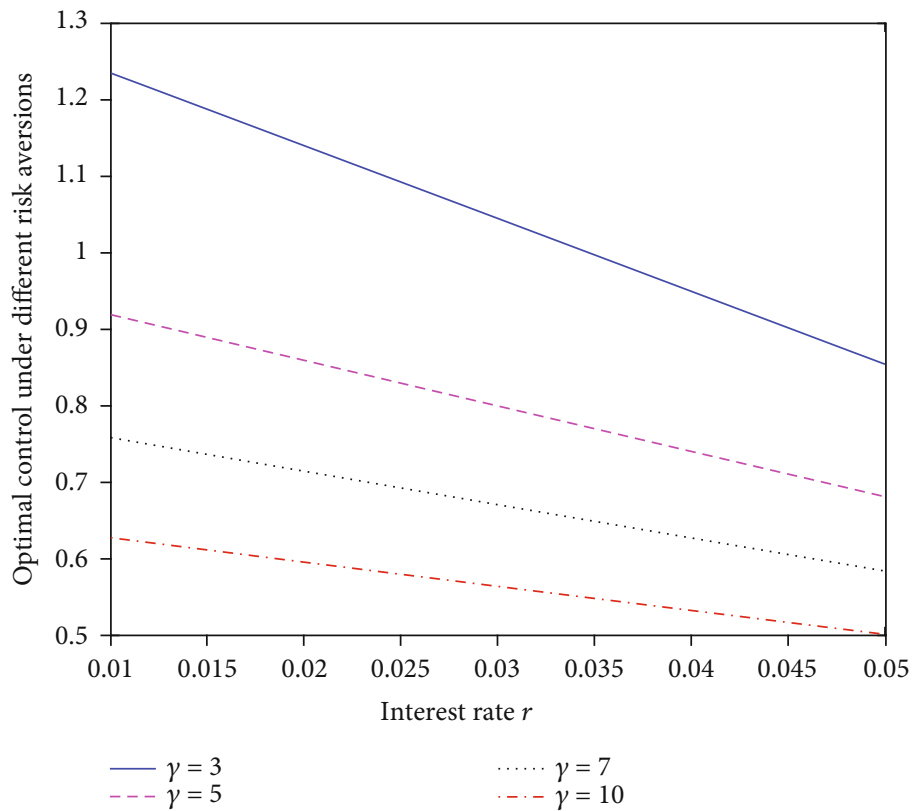

FIGURE 2: The relationship between $u^{*}$ and $r$ under different $\gamma$.

assets decreases, which means that the investor will allocate less money into the stock.

The optimal investment ratio versus the interest rate under different risk aversions is depicted in Figure 2. We could clearly see that the amount of money spent on the stock will decrease as the interest rate increases. This is consistent with our intuition. Also, we can find that under a small risk aversion, the change of interest rate $r$ causes more significant change in the amount of money spent on the stock, which means that under small risk aversion, the optimal control is more sensitive to the interest rate.
We also plot the total wealth of investors from the time when the interest rate is $r=0.01$ up to the existing time when $r=0.05$ for different SDRA value $\gamma(w)$. This is shown in Figure 3. The figure is also captured at the terminal time $T$ which only give a qualitative explanation. From the figure, we could see two trends: firstly, when risk aversions are small, the wealth will decrease as the interest increase. Since when the interest rate increases, we will spend less of the investor's wealth in the risky stock, as there is a chance that the general loss in the risky investment is higher than the bond return. Thus, the total wealth will decrease. On the other hand, when 


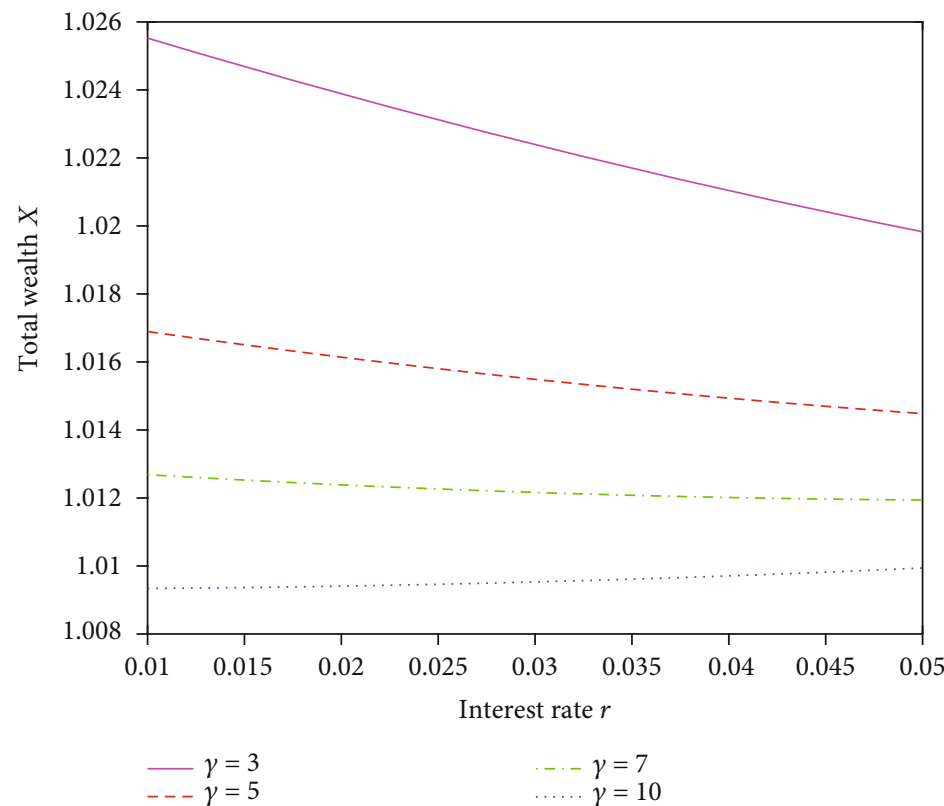

FIGURE 3: The relationship between total wealth and interest rate under different SDRAs.

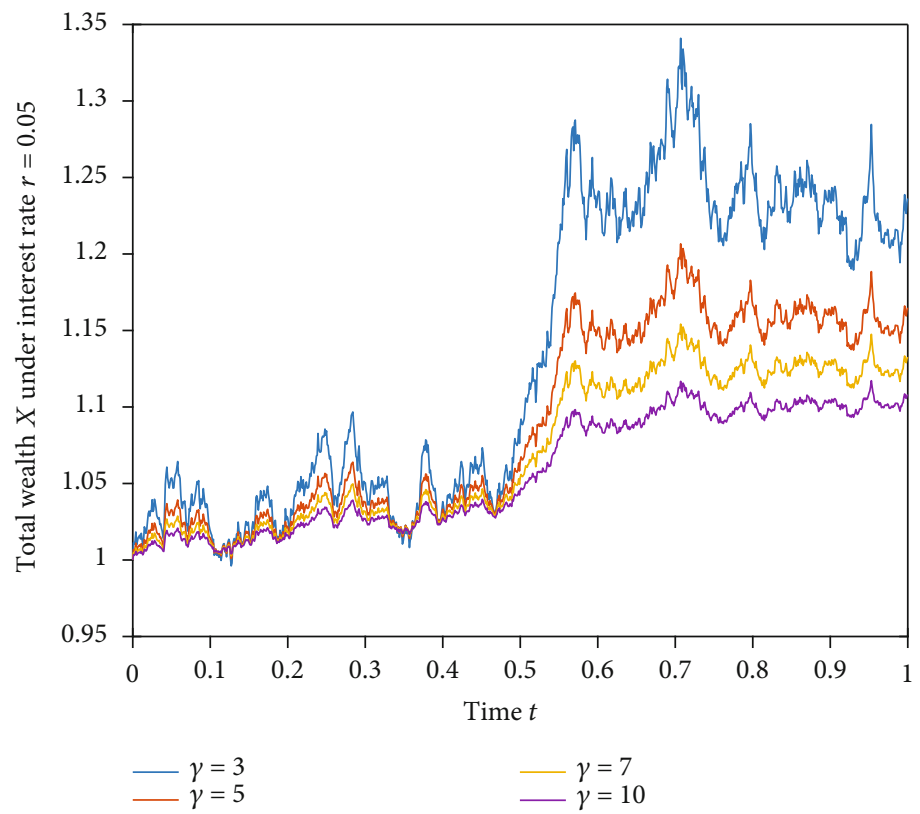

FIgURE 4: The relationship between total wealth and time under different SDRAs.

the risk aversions are large, we have a very limited investment in risky assets, so the total wealth will increase because of the relatively large amount of bonds return. But we can see that the increase is very mild, since the return of the risk-free bond will not be very high, which is reasonable.

For Figure 4, we show the investor's wealth evolution beginning from time $t=0$ to the terminal time $t=T$ under different SDRA value $\gamma(w)$. It is shown that generally, wealth processes all have an increasing trend. However, this increase has fluctuations due to the stochastic nature of the interest rate and the stock which are stochastic in the context of financial markets. Within a short time period, the wealth increases slowly with frequent fluctuations, and in the long run, the increasing trend will be more stable under the optimal control strategy. Moreover, when a CRRA value $\gamma$ is small, the investor has a relatively more fluctuated wealth process. When the $\gamma$ gets larger, the wealth process is relatively stable. This means that high risky assets sometimes bring us more return.

\section{Conclusions}

In this paper, we come up with an analytical solution for the portfolio optimization problem that contains a stochastic 
(short-term) interest rate. This problem is also governed by the mean-variance utility function with SDRA. We then proceed to use the Nash equilibrium for a subgame strategy to derive the equilibrium value function and also the equilibrium control policy and further provide analytical expressions for these. As a result, we present the closed-form solution for the associate portfolio selection model and analyse how the stochastic (short-term) interest rate and investor's risk aversion will affect the optimal control policy. This is done through a simplified financial setting that the investor has a "natural risk aversion." Finally, our numerical results show that in the presence of a stochastic (short-term) interest rate, the portfolio optimization problem is more economically sensible than the one when we assume a constant interest rate.

\section{Appendix}

\section{A. Derivation of the Theorem 2}

From the Hamilton-Jacobi-Bellman equation in [4], and the objective function (6), we can obtain

$$
Q\left(t, W_{t}, R_{t}\right)=\sup _{u \in U} J\left(t, W_{t}, R_{t}, U\right),
$$

and consequently have

$$
J\left(t, W_{t}, R_{t}, U\right)=E_{t, W_{t}, R_{t}}\left[F\left(y, W_{T}^{U}\right)\right]+G\left(y, E_{t, W_{t}, R_{t}}\left[W_{T}^{U}\right]\right),
$$

where the function $F$ and function $G$ have the forms described in (9).

When the time condition satisfies $l>t$, we have

$$
\begin{gathered}
J\left(l, W_{l}, R_{l}, U\right)=E_{l, W_{l}, R_{l}}\left[F\left(Y_{l}, W_{T}^{U}\right)\right]+G\left(Y_{l}, E_{l, W_{l}, R_{l}}\left[W_{T}^{U}\right]\right), \\
E_{l, W_{l}, R_{l}}\left[F\left(Y_{l}, W_{T}^{U}\right)\right]=f^{U}\left(l, W_{l}, R_{l}, Y_{l}\right), \\
E_{l, W_{l}, R_{l}}\left[W_{T}^{U}\right]=g^{U}\left(l, W_{l}, R_{l}\right) .
\end{gathered}
$$

Hence, the equation of (A.3) above can simply be written as,

$$
J\left(l, W_{l}, R_{l}, U\right)=f^{U}\left(l, W_{l}, R_{l}, Y_{l}\right)+G^{U}\left(Y_{l}, g^{U}\left(l, W_{l}, R_{l}\right)\right)
$$

On both sides of the equation, we apply expectations and then we can get,

$$
\begin{aligned}
E_{t, W_{t}, R_{t}}\left[J\left(l, W_{l}, R_{l}, U\right)\right]= & E_{t, W_{t}, R_{t}}\left[f^{U}\left(l, W_{l}, R_{l}, Y_{l}\right)+E_{t, W_{t}, R_{t}}\right. \\
& \cdot\left[G\left(Y_{l}, g^{U}\left(l, W_{l}, R_{l}\right)\right)\right],
\end{aligned}
$$

and combine this result into the definition of (A.2), we then have

$$
\begin{aligned}
& E_{t, W_{t}, R_{t}}\left[J\left(l, W_{l}, R_{l}, U\right)\right]=J\left(t, W_{t}, R_{t}, U\right)+E_{t, W_{t}, R_{t}} \\
& \quad \cdot\left[f^{U}\left(l, W_{l}, R_{l}, Y_{l}\right)\right]-E_{t, W_{t}, R_{t}}\left[F\left(y, W_{T}^{U}\right)\right] \\
& \quad+E_{t, W_{t}, R_{t}}\left[G\left(Y_{l}, g^{U}\left(l, W_{l}, R_{l}\right)\right)\right]-G\left(y, E_{t, W_{t}, R_{t}}\left[W_{T}^{U}\right]\right)
\end{aligned}
$$

From the condition of the iteration step by step, we obtain

$$
\begin{aligned}
E_{t, W_{t}, R_{t}}\left[F\left(y, W_{T}^{U}\right)\right] & =E_{t, W_{t}, R_{t}}\left[E_{l, W_{l}, R_{l}}\left[F\left(y, \mathrm{~W}_{T}^{U}\right)\right]\right] \\
& =E_{t, W_{t}, R_{t}}\left[f^{U}\left(l, W_{l}, R_{l}, y_{l}\right)\right],
\end{aligned}
$$

$E_{t, W_{t}, R_{t}}\left[W_{T}^{U}\right]=E_{t, W_{t}, R_{t}}\left[E_{l, W_{l}, R_{l}} W_{T}^{U}\right]=E_{t, W_{t}, R_{t}}\left[g^{U}\left(l, W_{l}, R_{l}\right)\right]$.

By substituting the results of (A.9) and (A.10) back into the equation of (A.8), we can get

$$
\begin{aligned}
& E_{t, W_{t}, R_{t}}\left[J\left(l, W_{l}, R_{l}, U\right)\right]-E_{t, W_{t}, R_{t}}\left[f^{U}\left(l, W_{l}, R_{l}, Y_{l}\right)\right] \\
& +E_{t, W_{t}, R_{t}}\left[f^{U}\left(l, W_{l}, R_{l}, y_{l}\right)\right]-J\left(t, W_{t}, R_{t}, U\right) \\
& \quad-E_{t, W_{t}, R_{t}}\left[G\left(Y_{l}, g^{U}\left(l, W_{l}, R_{l}\right)\right)\right] \\
& +G\left(y, E_{t, W_{t}, R_{t}}\left[g^{U}\left(l, W_{l}, R_{l}\right)\right]\right)=0 .
\end{aligned}
$$

Then,

$$
\begin{aligned}
& \sup _{u \in U}\left\{E_{t, W_{t}, R_{t}}\left[J\left(l, W_{l}, R_{l}, U\right)\right]-E_{t, W_{t}, R_{t}}\left[f^{U}\left(l, W_{l}, R_{l}, Y_{l}\right)\right]\right. \\
& \quad+E_{t, W_{t}, R_{t}}\left[f^{U}\left(l, W_{l}, R_{l}, y_{l}\right)\right]-J\left(t, W_{t}, R_{t}, U\right) \\
& \quad-E_{t, W_{t}, R_{t}}\left[G\left(Y_{l}, g^{U}\left(l, W_{l}, R_{l}\right)\right)\right] \\
& \left.\quad+G\left(y_{t}, E_{t, W_{t}, R_{t}}\left[g^{U}\left(l, W_{l}, R_{l}\right)\right]\right)\right\}=0 .
\end{aligned}
$$

Through our proposed problem (A.1) and the control law as defined in the traditional research, we find out that the control $U$ coincides with the equilibrium law $\widehat{u}$ in $[l, T]$, and then, we formulate the following,

$$
\begin{aligned}
J\left(l, W_{l}, R_{l}, \widehat{u}\right) & =Q\left(l, W_{l}, R_{l}\right), f^{U}\left(1, W_{l}, R_{l}, y\right) \\
& =f\left(l, W_{l}, R_{l}, y\right), g^{U}\left(l, W_{l}, R_{l}\right) \\
& =g\left(l, W_{l}, R_{l}\right) .
\end{aligned}
$$


Thus, the optimization problem of (A.12) can be given as

$$
\begin{aligned}
& \sup _{u \in U}\left\{E_{t, W_{t}, R_{t}}\left[Q\left(l, W_{l}, R_{l}\right)\right]-E_{t, W_{t}, R_{t}}\left[f\left(l, W_{l}, R_{l}, Y_{l}\right)\right]\right. \\
& \quad+E_{t, W_{t}, R_{t}}\left[f\left(l, W_{l}, R_{l}, Y_{l}\right)\right]-Q\left(t, W_{t}, R_{t}\right) \\
& \quad-E_{t, W_{t}, R_{t}}\left[G\left(Y_{l}, g\left(l, W_{l}, R_{l}, Y_{l}\right)\right)\right] \\
& \left.\quad+G\left(y_{t}, E_{t, W_{t}, R_{t}}\left[g^{U}\left(l, W_{l}, R_{l}, Y_{l}\right)\right]\right)\right\}=0 .
\end{aligned}
$$

Here, by using the following operator denotations for similarity

$$
\begin{aligned}
& E_{t, W_{t}, R_{t}}\left[Q\left(l, W_{l}, R_{l}\right)\right]-Q\left(t, W_{t}, R_{t}\right)=A^{u} Q, \\
& E_{t, W_{t}, R_{t}}\left[f\left(l, W_{l}, R_{l}, Y_{l}\right)\right] \\
& \quad=A^{u} f, E_{t, W_{t}, R_{t}}\left[f\left(l, W_{l}, R_{l}, y_{l}\right)\right]=A^{u} f^{y}, \\
& E_{t, W_{t}, R_{t}}\left[G\left(Y_{l}, g\left(l, W_{l}, R_{l}, Y_{l}\right)\right)\right] \\
& \quad=A^{u} G, G\left(y_{t}, E_{t, W_{t}, R_{t}}\left[g^{U}\left(l, W_{l}, R_{l}, Y_{l}\right)\right]\right) \\
& \quad=H^{u} g .
\end{aligned}
$$

We finally have developed the following extended HJB equation with stochastic volatility in the light of the work of [4],

$$
\begin{aligned}
& \sup _{u \in U}\left\{A^{u} V(t, r, w)-A^{u} f(t, r, w, w)+A^{u} f^{w}(t, r, w)\right. \\
& \left.-A^{u}(G * g)(t, r, w, w)+H^{u} g(t, r, w, w)\right\}=0,0 \leq t \leq T .
\end{aligned}
$$

\section{Data Availability}

The data used to support the findings of this study are available from the corresponding author upon request.

\section{Conflicts of Interest}

The authors declare that there is no conflict of interest regarding the publication of this paper.

\section{Acknowledgments}

This work was supported by the National Natural Science Foundation of China (Grants No.71974204 and No.71901222), the Humanities and Social Science Foundation of the Ministry of Education of China (Grant No. 17YJC630236), and "the Fundamental Research Funds for the Central Universities”, Zhongnan University of Economics and Law (No. 2722020JX005).

\section{References}

[1] H. Markowitz, "Portfolio selection," The Journal of Finance, vol. 7, no. 10, pp. 77-98, 1952.

[2] S. Li, C. Luong, F. Angkola, and Y. Wu, "Optimal asset portfolio with stochastic volatility under the mean-variance utility with state-dependent risk aversion," Journal of Industrial and
Management Optimization, vol. 12, no. 4, pp. 1521-1533, 2016.

[3] J. B. Detemple and Z. Fernando, "Asset prices in an exchange economy with habit formation," Econometrica, vol. 59, no. 6, pp. 1633-1657, 1991.

[4] T. Bjork, A. Murgoci, and X. Zhou, "Mean-variance portfolio optimization with state-dependent risk aversion," Mathematical Finance, vol. 24, no. 1, pp. 1-24, 2014.

[5] J. Yu and Y. Yuan, "Investor sentiment and the mean-variance relation «," Journal of Financial Economics, vol. 100, no. 2, pp. 367-381, 2011.

[6] F. E. Kydland and E. C. Prescott, "Rules rather than discretion: the inconsistency of optimal plans," The Journal of Political Economy, vol. 85, no. 1, pp. 473-492, 1997.

[7] H. R. Richardson, "A minimum variance result in continuous trading portfolio optimization," Management Science, vol. 35, no. 9, pp. 1045-1055, 1989.

[8] I. Bajeux-Besnainou and R. Portait, "Dynamic asset allocation in a mean-variance framework," Management Science, vol. 44, no. 11, pp. 79-95, 1998.

[9] D. Li and W. Ng, "Optimal dynamic portfolio selection: multiperiod mean-variance formulation," Mathematical Finance, vol. 10, no. 3, pp. 387-406, 2000.

[10] M. Dai, Z. XU, and X. Zhou, "Continuous-time Markowitz's model with transaction costs," SIAM Journal on Financial Mathematics, vol. 42, no. 1, pp. 96-125, 2010.

[11] H.-G. Xue, C.-X. Xu, and Z.-X. Feng, "Mean-variance portfolio optimal problem under concave transaction cost," Applied Mathematics and Computation, vol. 174, no. 1, pp. 1-12, 2006.

[12] A. E. B. Lim, "Quadratic hedging and mean-variance portfolio selection with random parameters in an incomplete market," Mathematics of Operations Research, vol. 29, no. 1, pp. 132161,2004

[13] T. R. Bielecki, H. Jin, S. R. Pliska, and X. Y. Zhou, "Continuous-time mean-variance portfolio selection with bankruptcy prohibition," Mathematical Finance, vol. 15, no. 2, pp. 213244,2005

[14] H. Yao, Y. Lai, Q. Ma, and H. Zheng, "Characterization of efficient frontier for mean-variance model with a drawdown constraint," Applied Mathematics and Computation, vol. 220, pp. 770-782, 2013.

[15] J. Xia, "Mean-variance portfolio choice: quadratic partial hedging," Mathematical Finance, vol. 15, no. 3, pp. 533-538, 2005.

[16] P. Christoffersen, S. Heston, and K. Jacobs, "The shape and term structure of the index option smirk: why multifactor stochastic volatility models work so well," Management Science, vol. 55, no. 12, pp. 1914-1932, 2009.

[17] S. L. Heston, "A closed-form solution for options with stochastic volatility with applications to bond and currency options," The Review of Financial Studies, vol. 6, no. 2, pp. 327-343, 1993.

[18] S. Li, Y. Zhou, Y. Wu, and X. Ge, "Equilibrium approach of asset and option pricing under Lévy process and stochastic volatility," Australian Journal of Management, vol. 42, no. 2, pp. 276-295, 2017.

[19] S. Liu, Y. Zhou, Y. Wu, and X. Ge, "Option pricing under the jump diffusion and multifactor stochastic processes," Journal of Function Spaces, vol. 2019, Article ID 9754679, 12 pages, 2019. 
[20] N. Vieille and J. W. Weibull, "Multiple solutions under quasiexponential discounting," Economic Theory, vol. 39, no. 3, pp. 513-526, 2009.

[21] S. M. Goldman, "Consistent plans," The Review of Economic Studies, vol. 47, no. 3, pp. 533-537, 1980.

[22] B. Peleg and M. E. Yaar, "On the existence of a consistent course of action when tastes are changing," The Review of Economic Studies, vol. 40, no. 3, pp. 391-401, 1973.

[23] R. A. Pollak, "Consistent planning," The Review of Economic Studies, vol. 35, no. 5, pp. 185-199, 1968.

[24] Z. Wu and Y. Zhuang, "Linear-quadratic partially observed forward-backward stochastic differential games and its application in finance," Applied Mathematics and Computation, vol. 321, pp. 577-592, 2018.

[25] P. Krusell and A. A. Smith, "Consumption-savings decisions with quasi-geometric discounting," Econometrica, vol. 71, no. 5, pp. 366-375, 2003.

[26] S. Basak and G. Chabakauri, "Dynamic mean-variance asset allocation," Review of Financial Studies, vol. 23, no. 8, pp. 2970-3016, 2010.

[27] T. Bjork and A. Murgoci, "A theory of Markovian timeinconsistent stochastic control in discrete time," Finance and Stochastics, vol. 18, no. 3, pp. 545-592, 2014.

[28] K. Chan, K. G. Andrew, A. F. Longstaff, and B. A. Sanders, “An empirical comparison of alternative models of the short-term interest rate," The journal of Finance, vol. 47, no. 3, pp. 1229-1237, 1992.

[29] R. C. Merton, “Theory of rational option pricing," The Bell Journal of Economics and Management Science, vol. 4, no. 1, pp. 141-183, 1973.

[30] O. Vasicek, "An equilibrium characterization of the term structure," Journal of Financial Economics, vol. 5, no. 2, pp. 177-188, 1977.

[31] J. C. Cox, J. E. Ingersoll, and S. A. Ross, "A theory of the term structure of interest rates," Econometrica, vol. 53, no. 2, pp. 385-407, 1985.

[32] J. E. Zhang, H. Zhao, and E. C. Chang, "Equilibrium asset and option pricing under jump diffusion," Mathematical Finance, vol. 22 , no. 3 , pp. 538-568, 2012. 